\title{
Interactive video game built for mask induction in pediatric patients
}

\author{
Samuel Rodriguez, MD • Jeremy H. Tsui $\cdot$ Samuel Y. Jiang, BA • \\ Thomas J. Caruso, MD
}

Received: 7 June 2017/ Accepted: 12 June 2017/Published online: 23 June 2017

(c) Canadian Anesthesiologists' Society 2017

\section{To the Editor,}

Induction of anesthesia is a stressful event for many pediatric patients. Preoperative distress has been correlated with postoperative agitation, negative behaviors, and increased postoperative pain. ${ }^{1}$ We recently introduced the "Sevo the Dragon" video game as a non-pharmacologic audiovisual intervention technique to reduce anxiety during induction. Consent to describe the case utilizing this technique was obtained from the patient's mother.

A six-year-old girl with a history of renal failure secondary to renal osteodystrophy and kidney transplant presented for a transplanted kidney biopsy. The patient had required physical restraint during a prior inhalational induction. On presentation for the biopsy, she appeared anxious, clutching her mother and communicating only through her. We discussed various anxiolytic options with the patient and her mother, including oral medications, video tablets, video projection through the Bedside Entertainment and Relaxation Theatre (BERT), ${ }^{2}$ and an interactive video game built on the BERT platform. The patient elected to try the interactive video game titled "Sevo the Dragon".

In the holding area, we set up a plastic screen $(24 \times 36$ inches) at the foot of the child's bed and displayed the game from a projector (Spro2, ZTE, USA) mounted on the bed's head rail. The patient selected a character from the three dragon options - red, blue, green, each with a different hat. She then chose from three food choices for

S. Rodriguez, MD · J. H. Tsui $(\bowtie) \cdot$ S. Y. Jiang, BA ·

T. J. Caruso, MD

Department of Anesthesiology, Perioperative, and Pain

Medicine, Lucile Packard Children's Hospital Stanford, Stanford

University School of Medicine, Palo Alto, CA, USA

e-mail: jeremy.tsui@yahoo.com the dragon to cook by breathing fire - birthday cake, tacos, pizza. The perioperative team controlled these choices through the mounted touchscreen projector. The game began in the preoperative area and then traveled with the patient to the operating room without interruption. The patient's mother helped hold the anesthesia mask to the patient's face facilitating anesthesia induction (Figure). The patient was engaged by the anesthesiologist who prompted her to breathe deeply to trigger the dragon to breathe fire. After the biopsy, the patient, parents, and medical staff reported high satisfaction with the compliant induction in contrast to her previously stormy experience.

This case illustrates successful application of an interactive video game on the BERT platform. Although audiovisual distraction for inhalational induction in pediatric cases has been described, ${ }^{3}$ most interactive video game systems are television- or tablet-based, with which children are generally familiar. In contrast, the use of BERT's large theatrical screen is supported by studies that detailed an association between the large size of the images and patients' emotional engagement with them. ${ }^{2}$ Other studies have shown the importance of novel vs familiar stimuli in capturing a patient's attention. ${ }^{4}$ In our case, both mother and patient were completely engrossed throughout the process. The mother later thanked the team, saying that the induction was "so cool."

The other unique feature of the game is that it not only distracts the child, it promotes cooperation with mask induction by encouraging deep breathing. By normalizing the breathing experience at induction via incorporating it into the game play, the patient displays a greater willingness to participate in the inhalational induction. A similar concept, the "blow up the balloon" method, has been employed for many years. ${ }^{5}$ The major drawbacks of the "balloon" technique are that it is difficult to keep the 

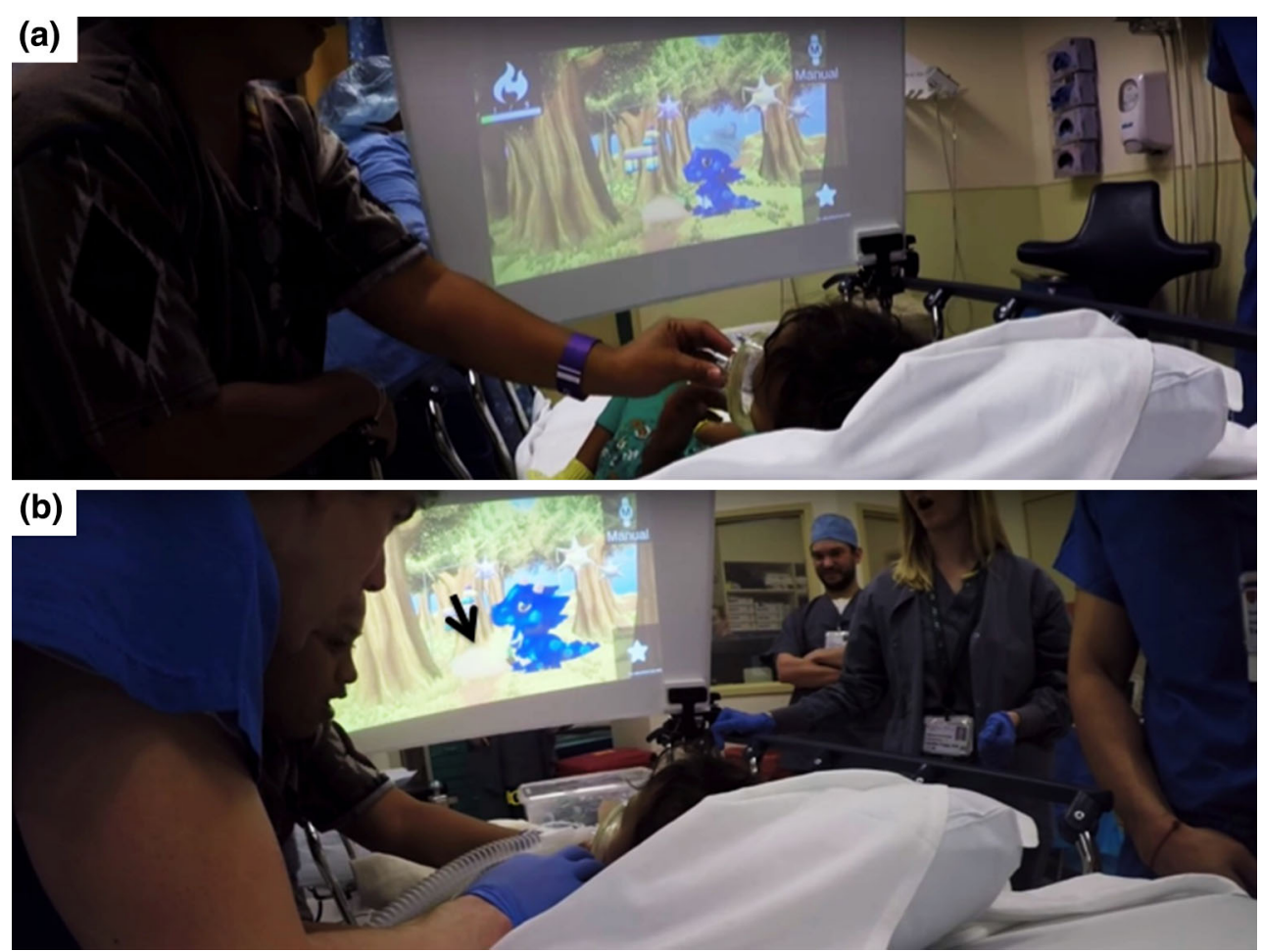

Figure "Sevo the Dragon" game integrated into the Bedside Entertainment and Relaxation Theatre (BERT) system for mask anesthesia induction. a) Deep breathing inhalation during game

child focused on blowing and watching the reservoir bag, which is attached to the intimidating anesthesia machine that is often poorly positioned ergonomically behind the patient. In contrast, our system combines the large screen and the interactive game projected in front of the child, which may be important factors for the efficacy of the "Sevo the Dragon" experience. The release date of this free android game is planned for August 1, 2017 on our website (chariot.stanford.edu).

Acknowledgements The authors thank Joseph Lang and the Traverse Foundation for their support in developing the BERT system and "Sevo the Dragon."

\section{Disclosures/conflict of interest None declared.}

Editorial responsibility This submission was handled by Dr. Gregory L. Bryson, Deputy Editor-in-Chief, Canadian Journal of Anesthesia. participation. b) "Sevo the Dragon" breathes fire (as shown by the arrow), emulating the patient's exhalation

\section{References}

1. Esteve R, Marquina-Aponte V, Ramírez-Maestre C. Postoperative pain in children: association between anxiety sensitivity, pain catastrophizing, and female caregivers' responses to children's pain. J Pain 2014; 15(157-68): e1.

2. Rodriguez S, Caruso T, Tsui B. Bedside Entertainment and Relaxation Theater: size and novelty does matter when using video distraction for perioperative pediatric anxiety. Paediatr Anaesth 2017; 27: 668-9.

3. Patel A, Schieble T, Davidson M, et al. Distraction with a handheld video game reduces pediatric preoperative anxiety. Paediatr Anaesth 2006; 16: 1019-27.

4. Mather E. Novelty, attention, and challenges for developmental psychology. Front Psychol 2013; 4: 491.

5. Malhotra SK, Dutta A, Kumar D. Play stickers: pediatric mask induction made easy. Anesth Analg 2001; 93: 1623. 